\title{
ZUR EFFIZIENZ DER MUTTERSPRACHENKOMPETENZ AUF DIE FREMDSPRACHENKOMPETENZ
}

\author{
Autorin
}

Aişe SEZIK 


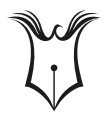

(C) Copyright 2021

Printing, broadcasting and sales rights of this book are reserved to Academician Bookstore House Inc. All or parts of this book may not be reproduced, printed or distributed by any means mechanical, electronic, photocopying, magnetic paper and/or other methods without prior written permission of the publisher. Tables, figures and graphics cannot be used for commercial purposes without permission. This book is sold with banderol of Republic of Turkey Ministry of Culture.

* This book has been generated from the doctoral dissertation which named as "Zur Effizienz der Muttersprachenkompetenz auf die Fremdsprachenkompetenz: Eine Didaktisch-Methodische Studie Für den Universitären Daf-Unterricht Bei Muttersprachlern des Türkischen" has written by Aişe SEZIK.

ISBN Page and Cover Design

978-625-8037-06-7 Typesetting and Cover Design by

Book Title

Zur Effizienz der Muttersprachenkompetenz auf die Fremdsprachenkompetenz Publisher Certificate Number 47518

Editor Printing and Binding

Prof. Dr. Ergün SERINDAĞ Vadi Printing Press

ORCID iD: 0000-0003-4226-9246

Author EDU018000

Aișe SEZIK

ORCID iD: 0000-0002-6633-7269

DOI

$* * *$

\section{Publishing Coordinator}

Yasin DİLMEN

\section{GENERAL DISTRIBUTION}

\section{Akademisyen Kitabevi A.Ş.}

Halk Sokak 5 / A

Yenişehir / Ankara

Tel: 03124311633

siparis@akademisyen.com 


\section{VORWORT}

Die Vollendung der vorliegenden Arbeit, verdanke ich vor allem einigen bedeutenden und hilfsbereiten Persönlichkeiten, die mich während der sorgfältigen Recherche und der Anfertigung zur Seite gestanden haben.

Zuerst möchte ich mich bei meinem Betreuer Prof. Dr. Ergün SERINDAĞ für seine wertvollen Vorschläge, klaren Orientierungen, Verbesserungen und natürlich für die Motivation bei der Themenfindung, herzlich bedanken, seinen besonderen Beitrag wei $\beta$ ich nämlich hochzuschätzen.

Ebenfalls bedanke ich mich bei unserem Abteilungsleiter Prof. Dr. Tahir BALCI, der in allen Angelegenheiten mit großer Toleranz auf mich zukam, meinem Jurymitglied Sabahattin ÇAM, der mich bei dem empirischen Teil dieser Arbeit mit seinen sachlichen Begründungen unterstützte und natürlich meinen lieben Arbeitskollegen Dr. phil. habil. Yasemin DARANCIK, Prof. Dr. Munise AKSÖZ, Asst. Prof. Dr. Cavidan ÇÖLTÜ IMREN, Lekt. Erdal KAÇAR, sowie Lektorin Dr. Çiğdem KURT, die durch hilfreiche Gespräche und Auskünfte Beiträge für die vorliegende Arbeit leisteten, sowie sich Zeit für mein Anliegen nahmen und mir positive Denkanstöße gaben.

Zweifelslos trug aber die Hauptlast der letzten Jahre meine Familie, deshalb möchte ich meinen persönlichen Dank meinem lieben Ehemann Mehmet, der mich während der Anfertigung dieser 
Arbeit in jeder Hinsicht emotional unterstützt, gefördert sowie in den letzten nervenaufreibenden Monaten immer zu Seite gestanden hat sowie, meinen Töchtern Ilayda und Adelya aussprechen, an deren Zeit ich stehlen musste, um die Vollendung dieser Arbeit realisieren zu können.

Der größte Dank geht jedoch an meine kleine Tochter Adelya, die schon im Mutterleib meinem ganzen Stress, während dieser Zeit, ausgesetzt war. 


\section{INHALTSVERZEICHNIS}

\section{KAPITEL I}

EINLEITUNG

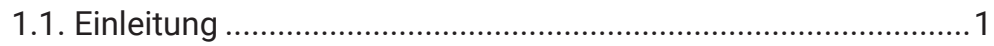

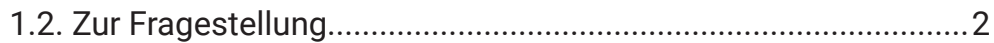

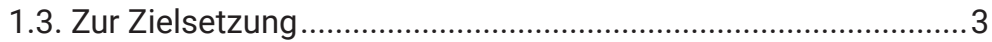

1.4. Zur Datenerhebung und Arbeitsmethode ................................ 4

1.5. Zum Aufbau der Arbeit................................................................... 5

KAPITEL II

INTERNATIONALE SCHULLEISTUNGSSTUDIEN

2.1. Internationale Schulleistungsstudien. 7

2.1.1. Die PISA-Studie als Beispiel für internationale Evaluierungen

2.1.2. Die Ergebnisse der PISA-Studie 2016 .................................

2.2. Einfluss der Ergebnisse auf die Fremdsprachenbildung .........13

KAPITEL III

GESELLSCHAFTLICHE WERTESTELLUNG DER SPACHE UND BEGRIFFSERKLÄRUNGEN: MUTTERSPRACHE, ERSTSPRACHE, ZWEITSPRACHE, FREMDSPRACHE

3.1. Was ist Sprache?

3.1.1. Bedeutung der Sprache. 16 
3.1.2. Was versteht man unter Muttersprache?

3.1.3. Was versteht man unter Erstsprache und Zweitsprache?

3.1.4. Was versteht man unter Fremdsprache? .........................20

3.2. Gegenüberstellung und Zusammenfassung der Begriffe........21

\section{KAPITEL IV}

\section{SPRACHERWERB}

4.1. Spracherwerb.

4.1.1. Erstsprachenerwerb und der Erwerb weiterer Sprachen ...23

4.1.2. Gesteuerter und ungesteuerter Erwerb .24

4.2. Zweitsprachenerwerb 26

4.2.1 Theorien zum Zweitsprachenerwerb .28

4.2.1.1. Die Kontrastivhypothese............................................28

4.2.1.2. Die Identitätshypothese ...............................................

4.2.1.3. Die Interlanguagehypothese .....................................33

4.2.1.4. Die Interdependenzhypothese ....................................35

4.2.1.5. Zusammenfassung der Theorien zum Zweitspracherwerb.

\section{KAPITEL V}

\section{ROLLE DER MUTTERSPRACHE IM FREMDSPRACHENUNTERRICHT}

5.1. Zur Rolle der Muttersprache für den Fremdsprachenerwerb ..39

5.1.1. Einflussfaktoren auf den Fremdsprachenerweb. 40

5.1.2. Der historische Überblick des Gebrauchs der Muttersprache im Fremdsprachenunterricht

5.1.2.1. Die Grammatik-Übersetzungsmethode 44 
5.1.2.2. Die Direkte Methode .45

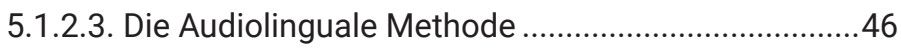

5.1.2.4. Die Audiovisuelle Methode.............................................

5.1.2.5. Die Kommunikative Didaktik ......................................47

5.1.3. Die unterschiedlichen Standpunkte der

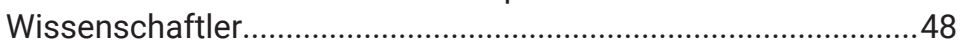

5.2. Die Muttersprache als Bezugsbasis.........................................53

5.2.1. Die Muttersprache bei der Bedeutungserschließung........54

5.2.2. Die Muttersprache bei der Wortschatzvermittlung.............55

5.2.3. Die Muttersprache als Textorientierung ............................57

5.2.4. Die Muttersprache als Mittel der Zeitersparnis ..................58

5.3. Ein Plädoyer für die Muttersprache ………………..................59

KAPITEL VI

METHODOLOGIE UND FORSCHUNGSDESINGN

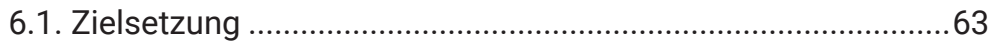

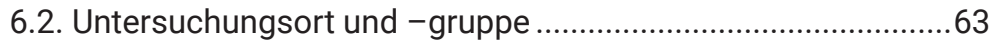

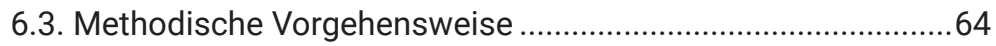

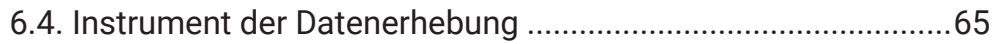

\section{KAPITEL VII}

\section{UNTERRICHTSPRAKTISCHE DURCHFÜHRUNGEN}

7.1. Unterrichtspraktischer Teil.

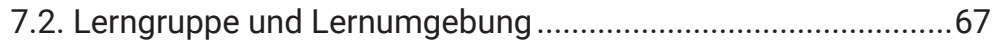

7.3. Ausarbeitung einer Unterrichtsstunde .....................................67 


\section{KAPITEL VIII \\ DATENAUSWERTUNG UND-INTERPRETATION}

8.1. Bewertung der Daten

8.1.1. Ergebnisse und Interpretation des ersten türkischen Tests (T1).

8.1.2. Ergebnisse und Interpretation des ersten türkischen Einstufungstests (T1) mit den Ergebnissen der deutschen Finalprüfung (D)

8.1.3. Ergebnisse und Interpretation des ersten türkischen Einstufungstests ( $\mathrm{T} 1$ ) mit den Ergebnissen des zweiten türkischen Einstufungstest (T2)

8.1.4. Ergebnisse und Interpretation des ersten türkischen Einstufungstests ( $\mathrm{T} 1$ ) mit den Ergebnissen des zweiten türkischen Einstufungstest (T2).................................................94

8.2. Zusammenfassung der Ergebnisse ......................................98

8.3. Statistische Analyse der Fragebogen .......................................99

8.3.1. Meinungsäußerungen der Studenten ...............................111

8.3.2. Interpretation der Ergebnisse der Experimentalgruppe ..115

8.3.3. Interpretation der Ergebnisse der Kontrollgruppe

KAPITEL IX

DISKUSSION UND SCHLUSSFOLGERUNG

9.1. Schlussfolgerung 119

9.1.1. Lerntheoretische Schlussfolgerung. 119

9.1.2. Didaktische Schlussfolgerung 121

9.1.3. Fremdsprachenpolitische Schlussfolgerung 122

LITERATURVERZEICHNIS 
nig Aufwand und Schwierigkeiten zu lernen. Da die Tatsache nun mal besteht, dass es allgemein beim Lernen von Fremdsprachen in der Türkei, trotz großen Aufwands, zum Scheitern kommt, muss sich dringend unsere Fremdsprachenpolitik ändern. Denn wie ein deutsches Sprichwort zum Ausdruck bringt: “Am Anfang zu heilen ist leichter als am Ende".

\section{LITERATURVERZEICHNIS}

Abdelilah-Bauer, B. (2008). Zweisprachig aufwachsen. Herausforderung und Chance für Kinder, Eltern und Erzieher. - München, C.H. Beck, München.

Ahvas, F.A. (2008). Welche Bedeutung hat der Erwerb der Muttersprache für den Erwerb der Zweitsprache. Magisterarbei Universität Wien.

Aðalsteinsdóttir, H. (2014). Alter und Motivation als Einflussfaktoren beim Fremdsprachenerwerb. Háskóli Íslands Hugvísindasvið Pýska. Verfügbar als Online-Dokument unter: https://skemman.is/bitstream/1946/17956/1/ Hugru\%CC\%81n\%20A\%C3\%B0alsteinsdo\%CC\%81ttir.pdf, gesehen am 01.02.2020.

Altrichter, H. (1998). Reflexion und Evaluation in Schulentwicklungsprozessen. In H. Altrichter, W. Schley \& M. Schratz (Hrsg.), Handbuch der Schulentwicklung (S. 263335). Innsbruck: Studienverlag.

Apeltauer, E. (1997). Grundlagen des Erst- und Fremdsprachenerwerbs. Fernstudieneinheit 15, S. 10-25, u.a.: Langenscheidt Verlag, Berlin

Apeltauer, E. (2003). Grundlagen des Erst- und Fremdsprachenerwerbs: eine Einführung (3. Auflage). Berlin, Wien (u.a.): Langenscheidt. (Fernstudieneinheit 15)

Atkinson, D. (1993). Teaching in the target language: a problem in the current orthodoxy. Volume 8. Verfügbar als Online-Dokument unter: https://doi. org/10.1080/09571739385200261, gesehen am 02.02.2020.

Attaviriyanupapi K. (2009). Hochdeutsch als Zweitsprache. Peter Lang Verlag Bern. Verfügbar als Online-Dokument: https://books.google.com.tr/ books? $\mathrm{id}=$ cjEjNOqYa68C\&pg =PA114\&lpg=PA1 14\&dq=Ausge $\%$ C3\%$\mathrm{BCbte}+\mathrm{Kritik}+\mathrm{an}+\mathrm{der}+$ Identit\%C3\% A4tshypothese\&source=bl\&ot s=3jH6hMwTsx\&sig=ACfU3U0G9nTH03M1zVPqQG29Z5dwcpRcVg\&hl=tr\&sa $=$ X\&ved =2ahUKEwi7n5yxoNPmAhWWShUIHXucDJYQ6AEwA3oECAUQAQ\#v=onepage\&q\&f=false, gesehen am 12.03.2019.

Balc1, T. (1993), Abriß der Türkisch-Deutschen kontrastiven Grammatik. Ein Lehrbuch für Studierende des Deutschen als Fremdsprache, Diyarbakır: Dicle Üniversitesi Yayınları, No:5Meisel, J.(1994). Bilingual First Language Acquisition. ISBN10 9027224714. Amsterdam

Baur, R.S. (2002). Schulischer Zweitsprachenerwerb bei Migrantenschülern 
Barkowski, H. (1980). Deutsch für ausländische Arbeiter. Gutachten zu ausgewählten Lehrwerken, Königstein, Kriptor.

Barkowski, H. (1995). Deutsch als Zweitsprach. In: Bausch, Karl- Richard/Christ, Herbert /Hüllen, Werner/ Krumm, Hans Jürgen. Hgg: Handbuch Fremdsprachenunterricht, S. 360-365, Tübingen.

Braun, M. (2019). Fünf Ideen einer Schülerin, wie Unterricht spannender werden könnte. Verfügbar als Online-Dokument: https://ze.tt/5-wege-wie-lehrende-ihren-schulunterricht-spannender-gestalten-koennen/, gesehen am 23.12.2019.

Brenska, P.(2011). Bilingualismus in einer polnisch-österreichischen Familie. Diplomarbeit, Universität Wien

Brockhaus (2010). Was ist Sprache? Verfügbar als Online- Dokument unter: www. lernhelfer.de, gesehen am 09.03.2017.

Bonfiglio, M. (2018). Die Rolle der Muttersprache "Deutsch" im Fremdsprachenunterricht. Ein Störfaktor oder eine Lernhilfe?, München, GRIN Verlag, https:// www.grin.com/document/431202

Burkard, C. (2005). Ergebnisorientierte Systemsteuerung: Konsequenzen für die externe Evaluation. In: G. Brägger, B. Bucher \& N. Landwehr (Hrsg.), Schlüsselfragen zur externen Schulevaluation (S. 79-109). Bern: h.e.p

Butzkamm,W.(1993). Psycholinguistik des Fremdsprachenunterrichts. Von der Muttersprache zur Fremdsprache, Tübingen/Basel: Francke

Butzkamm, W. (2002): Psycholinguistik des Fremdsprachenunterrichts: natürliche Künstlichkeit: von der Muttersprache zur Fremdsprache. Tübingen, Basel: A. Francke.

Butzkamm, W. (2003). We only learn language once. The role of the mother tongue in FL classrooms: Death of a dogma. December 2003,Language Learning Journal 28(1):S.29-39DOI: 10.1080/09571730385200181

Butzkamm, W. (2004). Forum Deutsch: Für Deutschlehrerinnen und Deutschlehrer in Kanada. Herausgeber: Canadian Association of Teachers of German. ISSN 0843-9829-X. Verfügbar als Online- Dokument: https://sites.ualberta.ca/ german/catg/fd2004.htm\#Butzkamm, gesehen am 14.02.2018.

Butzkamm, W. (2007). Native language skills as a foundation for foreign language learning. In book: Transcending boundaries. Essays in honor of Gisela Hermann-Brennecke, Publisher: LIT Verlag, Berlin

Butzkamm, W. ; Caldwell, J. A. W (2009). The Bilingual Reform. A Paradigm shift in Foreign Language Teaching. Gunter Narr Verlag Tübingen, ISBN: 978-38233-6492-4

Butzkamm, W. (2013). Neanderthal practice? On the teaching of grammar and the slaughtering of sacred cows. Conference: University of Poznan guest lecture at: Poznan, Poland.

Catford, J.C. (1967). A linguistic theory of translation: an essay in applied linguistics. Series: Language and Language Learnin, 8. Londan Oxford University Press

Çelebi, M. D. (2006). Türkiye'de anadil eğitimi ve yabancı dil öğretimi. Erciyes Üniversitesi Sosyal Bilimler Enstitüsü Dergisi, 2006/2 (21): 285- 307. 
Christ, I. (1992a).Bilinguale Züge im Sekundarbereich. In: Zernike College (Hrsg.), Grensoverschrijdend Talenonderwijs: Tweetaligheid in het Voortgezet Onderwijs, Euregionale Perspectieven; Grenzüberschreitender Sprachunterricht: Zweisprachige Sekundarstufe, Euregionale Perspektiven, Groningen: Zernike College, 49-66.

Christ, I.(1994). Het Duitse model. In: De Europese Onderwijspraktijk 02, Tweetalig onderwijs en versterkt talenonderwijs, Alkmaar: Stichting Europees Platform voor het Nederlandse Onderwijs, 103-118.

Christ, H. (2000). Von der Zielsprachendidaktik zur Didaktik der Mehrsprachigkeit? In: Helbig, Beate Kleppin, Karin/Königs, Frank G. (Hrsg): Sprachlehrforschungen im Wandel. Festschrift für Karl-Richard Bausch zum 60. Geburtstag. S.3-20.Tübingen

Corder, S.P. (1967). The significance of learner's errors. Julius Groos-Verlag. Volume 5, Seite:161-170 Heidelberg. Verfügbar als Online-Dokument: https://eric.ed. gov/?id=ED019903, gesehen am 26.12.2019.

Döbert, H., Klieme, E., Van Ackeren, I. et al. (2007). Vertiefender Vergleich der Schulsysteme ausgewählter PISA- Teilnehmerstaaten. Berlin. Verfügbar als Online-Dokument: http://www.bmbf.de/pub/pisa-vergleichsstudie.pdf, gesehen am 15.12.2017.

Dulay, H., Burt, M. (1974). Language Learning. Volume 24, S.37-45. Paper presented at the Annual TESOL Convention, Denver, Colorado.

Ebert, S., Köhne, J. \& Weinert, S. (2014). Mit Worten Türen öffnen: Sprache als Schlüsselkompetenz und Herausforderung für Forschung und Praxis. Universität Bamberg. Verfügbar als Online-Dokument: https://www.uni-bamberg.de/fileadmin/uni/verwaltung/presse/Dateien/2014/univers_Forschung_2014/10_Weinert.pdf, gesehen am 20.02.2018.

Edmondson, W., House, J. (2000). Einführung in die Sprachlehrforschung (2. Auflage). Tübingen, Basel: Francke (UTB für Wissenschaft 1697).

Edmondson, W.J., House, J. (2006). Einführung in die Sprachlehrforschung. A. Francke Verlag Tübingen und Basel. ISBN 10:3-8252-1697-7.

Faßbinder, K. (n.d). Grundlagen und Faktoren des Fremdsprachenerwerbs. Verfügbar als Online- Dokument unter: https://www.sprachenlernen24.de/blog/ voraussetzungen-lernen-fremdsprache/, gesehen am 01.02.2020.

Franssen, M. (2002). Bilingualer Unterricht in den Niederlanden und in Deutschland: eine Vergleichsstudie. Dissertation der Rheinisch-Westfälischen Technischen Hochschule Aachen.

Freudenstein, R. (1972): Linguistische Tips zum Lernen fremder Sprachen. In: GoetheIns titut (Hg.): Beiträge zu den Sommerkursen 1972, S. 43-55, München.

Fthenakis, W. (1985). Bilingual-bikulturelle Entwicklung des Kindes. Ein Handbuch für Psychologen, Pädagogen und Linguisten. München.

Glück, H. (1985). Zweisprachigkeit und Zweitsprachenerwerb. Ökonomische, politische und linguistische Argumente zu einer aktuellen sprachpolitischen Grundsatzdebatte. Deutsch lernen, 10. Seite: 15-42) 
Graf, P. (1987). Frühe Zweisprachigkeit und Schule. Empirische Grundlagen zur Erziehung von Minderheitenkindern. München: Hueber.

Grimm, H. (2000). Sprachentwicklung (Enzyklopädie der Psychologie. Bd. C/ III/3). Göttingen: Hogrefe.

Gurriai, A. (2015). PISA im Fokus. Verfügbar als Online-Dokument: https://www. oecd.org/berlin/themen/pisa-studie/PISA_2015_Zusammenfassung.pdf, gesehen am 16.02.2018.

Habersaat K, (1999). Hypothesen zum Zweitspracherwerb, München. GRIN Verlag, https://www.grin.com/document/72121

Hackl, K. (2013). Wissen und Einstellungen von Lehramtsstudierenden zu internationalen Schulleistungsstudien. Diplomarbeit, Universität Wien. Verfügbar als Online-Dokument: http://othes.univie.ac.at/27074/1/2013-03-19_0800857. pdf, gesehen am 16.02.2018.

Haidl, A. (2005). Zur Einsprachigkeit im Fremdsprachenunterricht: Kommentare, Fragen und Alternativen zu Wolfgang Butzkamms 13 Thesen.Acultad de Ciencias Sociales y de la Comunicación de la Universidad de Cádiz en Jerez de la Frontera. Verfübgar als Online- Dokument unter: https://www.researchgate. net/publication/304153121_Zur_Einsprachigkeit_im_Fremdsprachenunterricht_Kommentare_Fragen_und_Alternativen_zu_Wolfgang_Butzkamms_13_Thesen_2005, gesehen am 02.02.2020.

Hennon, E., Hirsh-Pasek, K. \& Golinkoff, R. M. (2000). Die besondere Reis vom Fötus zum spracherwerbenden Kind. In H. Grimm (Hrsg.), Sprachentwicklung (Enzyklopädie der Psychologie. Bd. C/III/3, S. 41-103). Göttingen: Hogrefe.

Hoppenstedt, G. (n.d). Die Muttersprache ist der Schlüssel für die zweite Sprache. Verfügbar als Online-Dokument unter: https://bildungsklick.de/fruehe-bildung/detail/die-muttersprache-ist-der-schluessel-fuer-die-zweite-sprache/, gesehen am 12.03.2019.

Hufeisen, B. (1991). Englisch als erste und Deutsch als zweite Fremdsprache:empirische Untersuchung zur fremdsprachlichen Interaktion. Frankfurt am Main: Verlag Peter Lang

İşigüzel, B. (2012). Der Einfluss der Erstsprache als ein Motivationsfaktor auf den Erfolg beim Fremdsprachenlernen. Dokuz Eylül Üniversitesi Sosyal Bilimler Enstitüsü Dergisi Cilt: 14, Say1: 2, Y1l: 2012, Sayfa: 53-86. ISSN: 1302-3284.

Jude, N. (2008). Zur Struktur von Sprachkompetenz. Dissertation Johann Wolfgang Goethe-Universität Frankfurt am Main. Verfügbar als Online- Dokument: https://d-nb.info/995928428/34, gesehen am 14.02.1018.

Kaiser, J.(2013). Zweit-/Fremdspracherwerb des Französischen und Code-Switching. Masterarbeit. München, GRIN Verlag. Verfügbar als Online-Domument: https://www.grin.com/document/232745

Karatay, H. (2007). İlköğretim Türkçe Öğretmeni Adaylarinin Okuduğunu Anlama Becerileri Üzerine Alan Araştirmasi. Gazi Üniversitesi Doktora Tezi, Ankara.

Keßler, J.U. (2006), Englischerwerb im Anfangsunterricht diagnostizieren: Linguistische Profilanalysen am Übergang von der Primarstufe in die Sekundarstufe I. Tübingen: Narr. 
Kınsız, M. (2001). Mutter-und Fremdspracherwerb in der Sprachentwicklung bei Kindern. Muğla Üniversitesi SBE Dergisi Bahar, Sayı 3. Verfügbar als Online-Dokument unter: https://dergipark.org.tr/en/download/article-file/217425, gesehen am 02.02.2020.

Klein, W. (1984). Zweitspracherwerb - Eine Einführung. Athenäum Verlag, Königstein, 1984.

Klein W. (2001). Typen und Konzepte des Spracherwerbs. Sprachwissenschaft, ein Reader, 902-924 (2010).

Kloiber, J. (2003). Was ist der Unterschied zwischen Muttersprache, Fremdsprache und Zweitsprache und in welchem Verhältnis stehen sie zueinander? Universität GH Essen. Hausarbeit.Verfügbar als Online-Dokument: https://www. grin.com/document/109781, gesehen am 21.02.2018.

Knapp-Potthoff, A. (1982), Fremdsprachenlernen und-lehren: eine Einführung in die Didaktik der Fremdsprachen vom Standpunkt der Zweitsprachenerwerbsforschung. Stuttgart: Kohlhammer.

Krestin, W. (2010). Wortschatzvermittlung im Deutsch als Fremdspracheunterricht unter Berücksichtigung des kognitiven Konzeptes des mentalen Lexikon. München, GRIN Verlag, https://www.grin.com/document/164472.

Kroker, B. (2018). Unterrichtseinstiege. Verfügbar als Online-Dokument: https:// www.betzold.de/blog/unterrichtseinstiege/, gesehen am 11.12.2019

Königs, F. G. (2000), Mehrsprachigkeit statt Sprachenlosigkeit. X. Lateinamerikanischer Germanistenkongress. Caracas 2000, 1-17 (Publikation auf CD).

Königs, F.G. (2015). Keine Angst vor Muttersprache - vor den (anderen) Fremdsprachen aber auch nicht! Überlegungen zum Verhältnis von Einsprachigkeit und Zweisprachigkeit im Fremdsprachenunterricht. Zeitschrift für Interkulturellen Fremdsprachenunterricht Didaktik und Methodik im Bereich Deutsch als Fremdsprache ISSN 1205-6545 Jahrgang 20, Nummer 2. Verfügbar als Online-Dokument unter: file://C:/Users/toshiba/Downloads/756-1514-1-SM. pdf, gesehen am 03.02.2020.

Kunštek, V. (2015). Muttersprache beim Fremdsprachenlernen. Diplomarbeit. Zagreb. Verfügbar als Online-Dokument unter: http://darhiv.ffzg.unizg.hr/id/ eprint/5279/1/Diplomski\%20rad_Muttersprache\%20beim\%20Fremdsprachenlernen.pdf, gesehen am 30.01.2020.

Lehmann, C. (2013). Sekundärsprachenerwerb. Verfügbar als Online-Dokument: https://www.christianlehmann.eu/ling/elements/index.html?https://www. christianlehmann.eu/ling/elements/sekundaerspracherwerb.html, gesehen am 25.06.2019.

Lipińska, E., Seretny, A. (2006). Z zagadnień dydaktyki języka polskiego jako obcego. Metodyka nauczania. - Kraków: Universitas, S. 19-26.

Majzlan, V. (2013). Polnischsprachige Kinder in Wien: Sprachliche Besonderheiten in der Kontaktsituation.Diplomarbeit. Wien.

Martín Peris, E. (2008). Diccionario de téminos clave de ELE, Alcobendas (Madrid): Sgel, Soc. Gen. Española de Librería. 
Mäsch, N. (1994). Das deutsche Modell bilingualer Erziehung in den Sekundarstufen I und II. In: De vreemde taal als instructietaal: problemen, prioriteiten en perspectieven. Conferentie tweetalig onderwijs, Utrecht, 16 februari 1994, 27-38.

Meier, P. (2006). Der bewusste Einsatz der Muttersprache im Fremdsprachenunterricht. München, GRIN Verlag, verfügbar als Online- Dokument: https://www. grin.com/document/168596, gesehen am 14.02.2018.

Meijer, T. (1974). The global-bilingual and the audio-visual approach to meaning conveyance. A comparison of two methods for firs-year secondary French teaching. Amsterdam: Vrije Universität)

Meyer, H. (2008). The pedagogical implications of L1 use in the L2 classroom. Maebashi Kyoai Gakuen College Ronsyu, 8: 147-159, verfügbar als Online-Dokument unter: www.kyoai.ac.jp/college/ronshuu/no-08/meyer1.pdf (03.04.2017).

Meyers Enzyklopädisches Lexikon (1976), Band 16

Neuner, G. ;Hunfeld, H. (1993): Methoden des fremdsprachlichen Deutschunterrichts: eine Einführung. Fernstudieneinheit 4. Berlin: Langenscheidt.

Neuner, G. (2003). Mehrsprachigkeitskonzept und Tertiärsprachendidaktik.In: Mehrsprachigkeitskonzept - Tertiärsprachenlernen - Deutsch nach Englisch von Britta Hufeisen; Gerhard Neuner. ISBN 92-871-5146-6, Europarat.

Oecd.org (2018). Was ist Pisa? Verfügbar als Online-Dokument: http://www.oecd. org/berlin/themen/pisa-studie/, gesehen am 16.02.1018.

Olariu, A. (2007). Individuelle Mehrsprachigkeit und begriffliche Gegenüberstellung von: Erstsprache, Muttersprache, Zweitsprache und Fremdsprache. Philologica Jassyensia Nr.2. Verfügbar als Online-Dokument: http://www.diacronia.ro/ ro/indexing/details/A758/pdf, gesehen am 26.02.2018.

Oksaar, E. (2003): Zweitspracherwerb. Wege zur Mehrsprachigkeit und zur interkulturellen Verständigung. Kohlhammer, Stuttgart.

ÖSYM (2013). Yüksek Öğretime Geçiş Sınavı Soruları. Verfügbar als Online- Dokument unter: https://www.scribd.com/document/165221008/24-03-2013-YGS, gesehen am 18.05.2017.

Petek, M. (2013). Spracherwerb in Erst- und Zweitsprache. Gastvortráge an der Masaryk Universitát in Brlinn, verfügbar als Online-Dokument: https://is.muni. cz/el/1441/jaro2015/N2BP_4DID/Spracherwerb_-_M.Petek.pdf, gesehen am 26.12.2019.

Petrović, E. (1988). Teorija nastave stranih jezika. Zagreb: Školska knjiga

Pienemann, M. (1998). Language processing and second language de velopment: Processability theory. Amsterdam/Philadelphia.

Pinar, Y. (2015). Interaktion, Emotion und Sprache: Unter besonderer Berücksichtigung des Zweitsprachenerwerbs im Kindergarten.Lit-Verlag, Berlin. Verfügbar als Online-Dokument: https://books.google.com.tr/books?id=YGadDQAAQBAJ\&pg=PA39\&lpg=PA39\&dq=kritik+an+der+identitaetshypothese\&source=bl\&ots=e312MTxA9Y\&sig=ACfU3U2KTzk4Shn02LYIA- 
6tiMvt86skPlg\&hl=tr\&sa=X\&ved=2ahUKEwjNiKif-tLmAhVQaVAKHRPjAw0Q6AEwBHoECAgQAQ\#v=onepage\&q=kritik\%20an\%20der\%20 identitaetshypothese\&f=false, gesehen am 20.01.2019.

Plieger, P. (2006). Struktur und Erwerb des bilingualen Lexikons. Konzepte für die mediengestützte Wortschatzarbeit. LIT-Verlag, Berlin. Verfügbar als Online-Dokument unter: https://books.google.com.tr/books?id=Fosrs7NuVEUC\&pg $=$ PA147\&lpg $=$ PA147\&dq $=$ Die + Muttersprache + bei + der + Wort schatzvermittlung \&source $=$ bl\&ots $=$ ctzM8hN94Y \&sig $=$ ACfU3U2JGGhwspboGNsOHCh4zv3oMul7qQ\&hl=tr\&sa=X\&ved=2ahUKEwjs_oDTnLrnAhWFycQBHS4oBE0Q6AEwAHoECAkQAQ\#v=onepage\&q=Die\%20 Muttersprache\%20bei\%20der\%20Wortschatzvermittlung\&f=false, gesehen am 05.02.2020.

Prenzel, M. \& Seidel, T. (2010). Bildungsmonitoring und Evaluation. In C. Spiel, B. Schober, P. Wagner \& R. Reimann (Hrsg.), Bildungspsychologie (S. 329-345). Göttingen: Hogrefe.

Prigl, E. (2010). Die Rolle der Erstsprache im Fremdsprachenunterricht" Theorie und Praxis hinsichtlich der Verwendung der Erstsprache der SchülerInnen seitens der Lehrkräfte. Bachelorseminararbeit. Universität Wien. Verfügbar als Online-Dokument unter: https://www.academia.edu/6485338/_Die_Rolle_ der_Erstsprache_im_Fremdsprachenunterricht_Theorie_und_Praxis_hinsichtlich_der_Verwendung_der_Erstsprache_der_Sch\%C3\%BClerInnen_seitens_der_Lehrkr\%C3\%A4fte, gesehen am 02.02.2020.

Renner, J. (2016). Die Bedeutung der Sprache für das soziale Handeln und die kulturelle Identität von Gesellschaften. Universität Konstanz. Verfügbar als Online-Dokument: https://www.hausarbeiten.de/document/343714, gesehen am 20.02.2018.

Roche, J. (2008): Fremdsprachenerwerb, Fremdsprachendidaktik. Tübingen: A. Francke.

Rothweiler, M., Meibauer, J., Demske J., Geilfuß, J., Pafel, J.W., Ramers, K.H., Steinbach, M. (2015). Einführung in die germanistische Linguistik. Springer-Verlag Berlin Heidelberg.Online ISBN978-3-476-05424-1

Sagir M. (2006). Der Zweitspracherwerb von Kindern mit Migrationshintergrund, GRIN Verlag, München. Verfügbar als Online-Dokument: https://www.grin. com/document/110192, gesehen am 25.12.2018.

Salman, U.A. (2016). PISA 2015'teki düşüşün sebebi ne? Verfügbar als Online-Dokument: http://www.aljazeera.com.tr/al-jazeera-ozel/pisa-2015teki-dususun-sebebi-ne, gesehen am 19.02.2018.

Sastri, H.N.L. (1970). The Bilingual Method of Teaching English - An Experiment. Sage Journals. Volume: 1 issue: 2, page(s): 24-28

Saussures De, F. (1967): Grundfragen der allgemeinen Sprachwissenschaft. (Kap. VI: „Wiedergabe der Sprache durch die Schrift“), Berlin 2, Seite: 27,28.

Scharff Rethfeldt,W. (2013). Kindliche Mehrsprachigkeit. DOI: 10.1055/b-003457784, verfügbar als Online-Dokument: https://www.thieme-connect.de/ 
products/ebooks/pdf/10.1055/b-0034-57784.pdf\#, gesehen am 26.12.2019. Schröder, U. (2007). Holistische und integrative Tendenzen in der Zweitspracherwerbsforschung. Universidad Nacional de Colombia - Facultad de Ciencias Humanas - Bogot, verfügbar als Online-Dokument: https://www.academia. edu/35002523/HOLISTISCHE_UND_INTEGRATIVE_TENDENZEN_IN_ DER_ZWEITSPRACHERWERBSFORSCHUNG, gesehen am 25.12.2019.

Schwerdfeger, I.C. (2001). Gruppenarbeit und innere Differenzierung. Ferstudieneinheit. Berlin Langenscheidt.

Selinker L. (1972). The interlanguage hypothesis extended to children. Language learning VOL. 25, NO. 1. University of Washington. Verfügbar als Online-Dokument: https://www.researchgate.net/profile/Merrill_Swain/publication/229551148_The_Interlanguage_Hypothesis_Extended_to_Children/ links/5a524d7e458515e7b72ca8dc/The-Interlanguage-Hypothesis-Extended-to-Children.pdf.

Serindağ, E. (2003). Zur Didaktik und Methodik der Ausnutzung des Englischen als erster Fremdsprache im Unterricht "Deutsch als zweite Fremdsprache" bei Muttersprachlern des Türkischen. Yayınlanmış doktora tezi. Çukurova Üniversitesi Sosyal Bilimler Enstitüsü, Adana.

Siebert-Ott, G. (2003). Muttersprachedidaktik,Zweitsprachendidaktik, Fremdsprachendidaktik, Multilingualität. In: Bredel,U.;Günter,H.;Klotz,P.;Ossner,J.;Siebert- Ott,G. (Hrsg.):Didaktik der deutschen Sprache - ein Handbuch,Band 1, 2. Auflage. Paderborn, (30-41). Verfügbar als Online-Dokument: http:// www.beck-shop.de/fachbuch/leseprobe/9783825282356_Excerpt_001.pdf, gesehen am 27.02.2018.

Spolsky, B. (1989): Conditions for second language learning: Introduction to a general theory. Oxford: Oxford University Press. Verfübar als Online-Dokument unter: http://www.slm.unihamburg.de/iaas_slf/Materialien/067.pdf (abgerufen am 17.03.2014)

Stamm, M. (2003). Evaluation und ihre Folgen für die Bildung. Eine unterschätzte pädagogische Herausforderung. In: Internationale Hochschulschriften Bd. 419. Münster: Waxmann Verlag.

Unruh T., Petersen S. (2002). Guter Unterricht. AOL-Verlag. ISBN-10: 389111639X

ISBN-13: 978-3891116395. Verfügbar als Online-Dokument: https://www.guterunterricht.de/unterrichtsphasen, gesehen am 23.12.2019.

Vollmer, H. J. (2000b). Förderung des Spracherwerbs im bilingualen Sachfachunterricht. In: Bach, Gerhard \& Susanne Niemeier (Hrsg.), Bilingualer Unterricht. Grundlagen, Methoden, Praxis, Perspektiven. Frankfurt am Main: Lang, 139158.

Wahrig, G. (n.d). Deutsches Wörterbuch. Bertelsmann Lexikon Verlag GMBH. München. İSBN-3-577-10446-5

Weller, F.R. (1981). Formen und Funktion der Übersetzung im Fremdsprachenunterricht. Beispiel Französisch. S.233-296, Frankfurt am Main, Diesterweg.

Wikipedia (2019). Haus des Geldes. Verfügbar als Online-Dokument: https:// 
de.wikipedia.org/wiki/Haus_des_Geldes, gesehen am 11.12.2019

Wode, H.(1993). Psycholinguistik: Eine Einführung in die Lehr- und Lernbarkeit von Sprachen. Hueber, Ismaning,

Wojnesitz, A. (2009). Sprachbewusstsein und Einstellungen zur Mehrsprachigkeit an Wiener AHS im Kontext von Migration. Dissertation Universität Wien. Verfügbar als-Online Dokument: http://othes.univie. ac.at/4709/1/2009-04-04_9105632.pdf, gesehen am 23.02.2018.

Torlak, M. (2010). Yabancı dili öğrenmede ana dili bilmenin etkisi. Kandildergisi sayı1. Verfügbar als Online-Dokument unter: http://www.kandildergisi. com/2010/10/yabanci-dili-ogrenmede-ana-dili-bilmenin-etkisi, gesehen am 28.02.2017

Wunderlich, D. (2002). Was ist Sprache? Einführung. Vorlesung Universität Düsseldorf. Verfügbar als Online-Dokument: https://user.phil.hhu.de/ wdl/Wasist-1.pdf, gesehen am 04.01.2018.

Zybatow G. (2007). Theorien des Zweitspracherwerbs. Universität Leipzig. Verfügbar als Online-Dokument: https://is.muni.cz/el/ped/jaro2014/N2BP_4DID/ Theorien_des_Zweitspracherwerbs_-_Handout.pdf?lang=en, gesehen am 26.12.2019. 